\title{
Analyse experimentale de l'écoulement dans la zone axiale des canaux adducteurs des turbines-hélices
}

\section{Experimental analysis of the flow in the axial region of propeller-turbine admission ducts}

\author{
R. Bettocchi et G. Cantore*, \\ L. Magri et M. Ubaldi**
}

* Professeurs associés à l'Istituto di Macchine de l'Université de Bologna

** Ingénieurs au bureau de recherche et développement, Hydroart S.p.A. Milano

\section{Introduction}

La distribution des vitesses en amont de la roue en influence le rendement et le comportement à la cavitation, d'où l'importance d'étudier l'allure de l'écoulement dans l'espace entre distributeur et roue et en particulier, pour les turbines-hélices, dans la zone axiale du conduit dans laquelle on place la roue.

On présente ici les résultats de quelques relevés expérimentaux de vitesse effectués dans des conduits adducteurs de turbines-hélices à différentes ouvertures

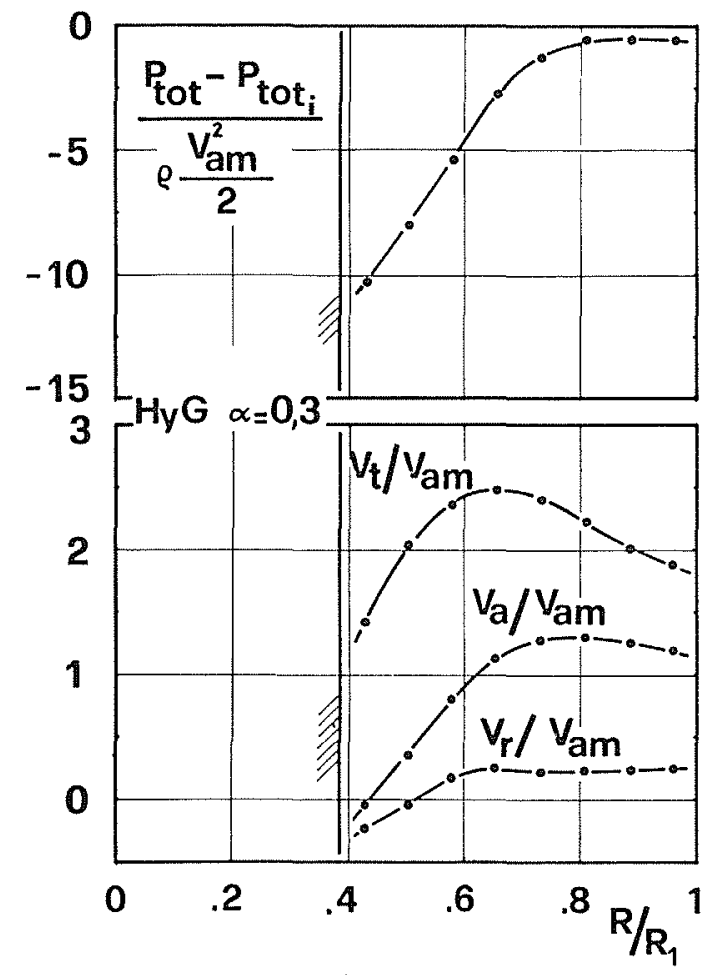

Figure 1 - Exemple de répartition des vitesses et pressions totales aux petites ouvertures du distributeur. du distributeur, en concentrant l'attention sur les phénomènes qui se vérifient aux petites ouvertures. En effet, lorsque l'ouverture du distributeur diminue, on assiste près du moyeu (voir fig. 1) à une modification de la distribution radiale de vitesse tangentielle avec formation d'une zone à tourbillon presque forcé, accompagnée d'une diminution et parfois l'inversion de la vitesse axiale et des vitesses radiales dirigées vers l'axe du conduit, on constate aussi la diminution de la pression totale, signe de dissipation visqueuse.

Ce phénomène est analogue à celui qui se remarque au centre de tuyaux dans lesquels des composantes tangentielles de vitesse ont été crées par un distributeur placé en position radiale ou axiale [1], [2], [3].

Dans les turbines axiales ce phénomène provoque une baisse du rendement et donne lieu à des tourbillons cavitants qui se détachent près du moyeu et, en s'enroulant en un mouvement helicoïdal, passent au travers de la roue en créant des vibrations et des bruits dans le fonctionnement [4].

\section{Relevés expérimentaux}

Les relevés expérimentaux ont été effectués, d'une part, en eau, dans le canal adducteur d'un modèle de turbine-hélice situé dans un des laboratoires de la Société Hydroart (Hy) et, d'autre part, en air, dans un conduit cylindrique à section annulaire alimenté par un distributeur radial à aubes planes [5] situé dans le laboratoire de l'Institut de Machines de l'Université de Bologne (IMB).

Sur le modèle Hy d'origine dessiné en trait continu à gauche de l'axe sur la fig. 2, on a effectué des mesures de vitesse avec et sans roue dans les sections HyE et HyF.

En outre, on a relevé, en particulier aux petites ouvertures du distributeur, l'évolution axiale de l'allure des vitesses dans le canal hydraulique sans roue et ayant 


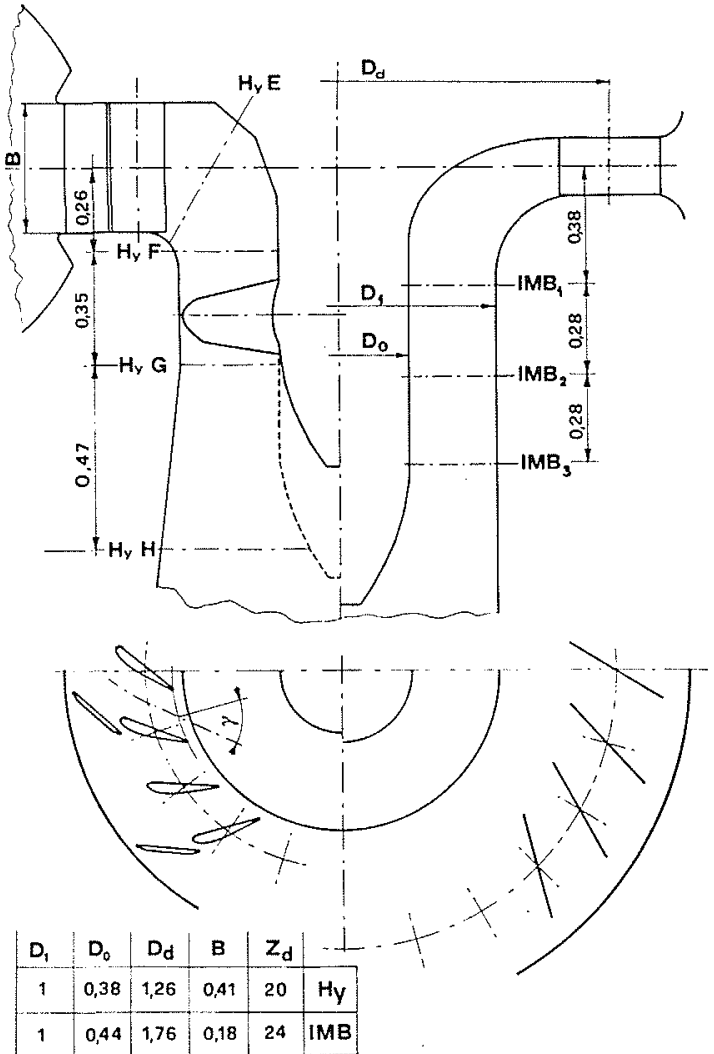

Figure 2 - Géométrie des deux canaux et sections de mesure.

la zone axiale prolongée par rapport à la configuration d'origine, comme indiqué par la ligne en pointillé de la fig. 2. Ces mesures ont été effectuées dans les sections $\mathrm{HyG}$ et $\mathrm{HyH}$.

Indépendamment, on a effectué des relevés expérimentaux dans le conduit IMB, représenté à droite de la fig. 2, dans les sections IMB 1, IMB2 et IMB3.

Ce conduit, alimenté en chambre libre, est muni d'un distributeur simplifié formé d'aubes directrices planes placées en zone d'écoulement essentiellement radiale(1). La hauteur des aubes est plus petite et la distance "axe des tourillons-axe de la machine" est plus grand que dans le cas du distributeur Hy.

Les relevés expérimentaux effectués dans les deux canaux ont été analysés conjointement pour mettre en évidence les analogies dans l'évolution des distributions de vitesse en fonction de l'ouverture du distributeur.

Les mesures Hy ont été effectuées avec une sonde prismatique à 5 trous United Sensor et les relevés IMB avec sonde sphérique à 5 trous.

La gamme des nombres de Reynolds explorés s'étend de $2.10^{5}$ à $4.10^{5}$ pour IMB et de $3.10^{5}$ à $9.10^{5}$ pour Hy.

(1) Ce distributeur avait été réalisé dans le seul but de conférer à la veine fluide des prérotations voulues dans le cadre de recherches sur l'écoulement tourbillonnant effectuées à l'Institut de Machines de l'Université de Bologne.
Evolution de l'écoulement dans une section de mesure en zone axiale en fonction de l'ouverture du distributeur

Sur les figures 3 et 4 on a indiqué, pour différentes ouvertures du distributeur, les répartitions, respectivement, dans les sections IMB2 et $\mathrm{HyG}$, des composantes axiales et tangentielles de la vitesse rapportées à la vitesse axiale moyenne. Les deux sections IMB2 et $\mathrm{HyG}$ ont été choisies parce qu'elles étaient les plus proches parmi toutes celles explorées dans les deux canaux.

Les figures 3 et 4 montrent avant tout que les distributions des composantes axiales et tangentielles de la vitesse se modifient de façon presque analogue dans les deux canaux lorsque l'ouverture du distributeur diminue.

$\mathrm{Au}$ voisinage du moyeu, la vitesse axiale augmente d'abord légèrement, puis diminue jusqu'à des valeurs nulles ou négatives créant ainsi une zone d'eau morte ou de recirculation.

Aux ouvertures élevées, la répartition de vitesse tangentielle s'approche d'autant plus d'une configuration à tourbillon libre autour de l'axe du canal que les aubes du distributeur se trouvent en zone à écoulement radial (IMB). Aux petites ouvertures du distributeur, prés du moyeu, dans une zone centrale dont les dimensions peuvent être comparées à celles du noyau central à vitesse axiale faible ou négative, il existe une distribution des vitesses tangentielles à tourbillon presque forcé qui confine la zone à tourbillon presque libre dans une couronne annulaire se rapprochant du manteau lorsque l'ouverture diminue [5].

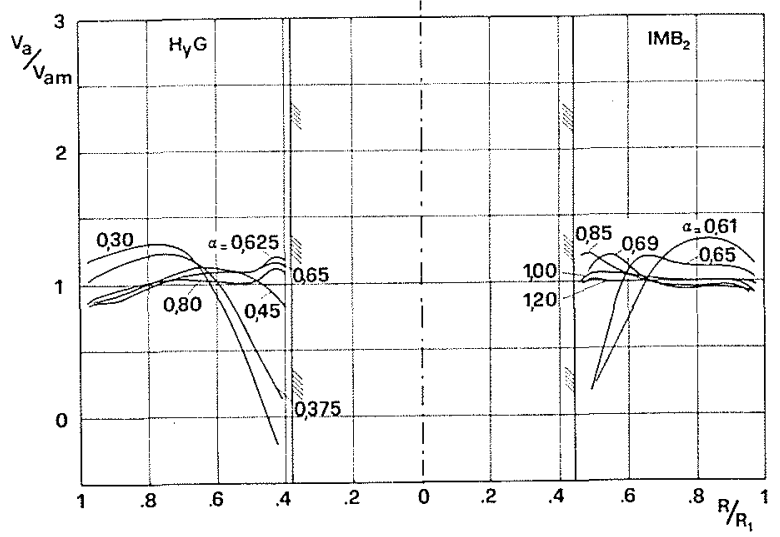

Figure 3 - Evolution de la répartition des vitesses axiales au fur et à mesure que l'ouverture du distribu teur varie.

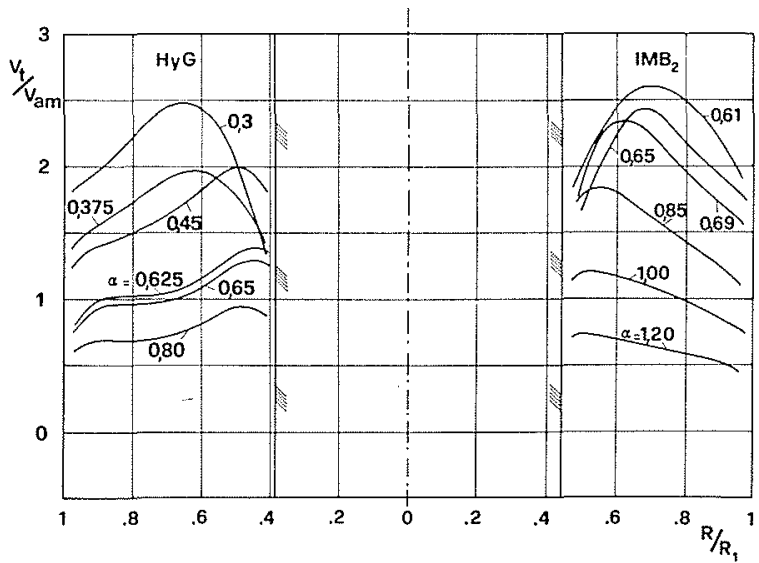

Figure 4 - Evolution de la répartition des vitesses tangentielles au fur et à mesure que l'ouverture du distributeur varie. 


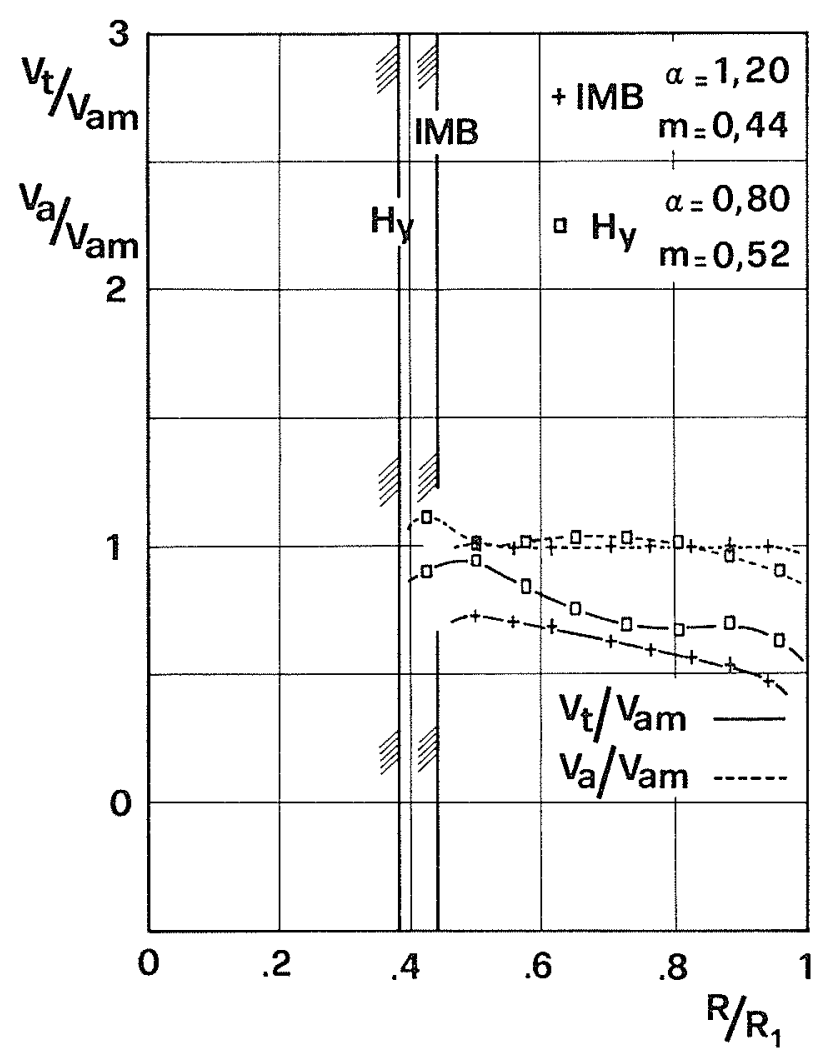

Figure 5

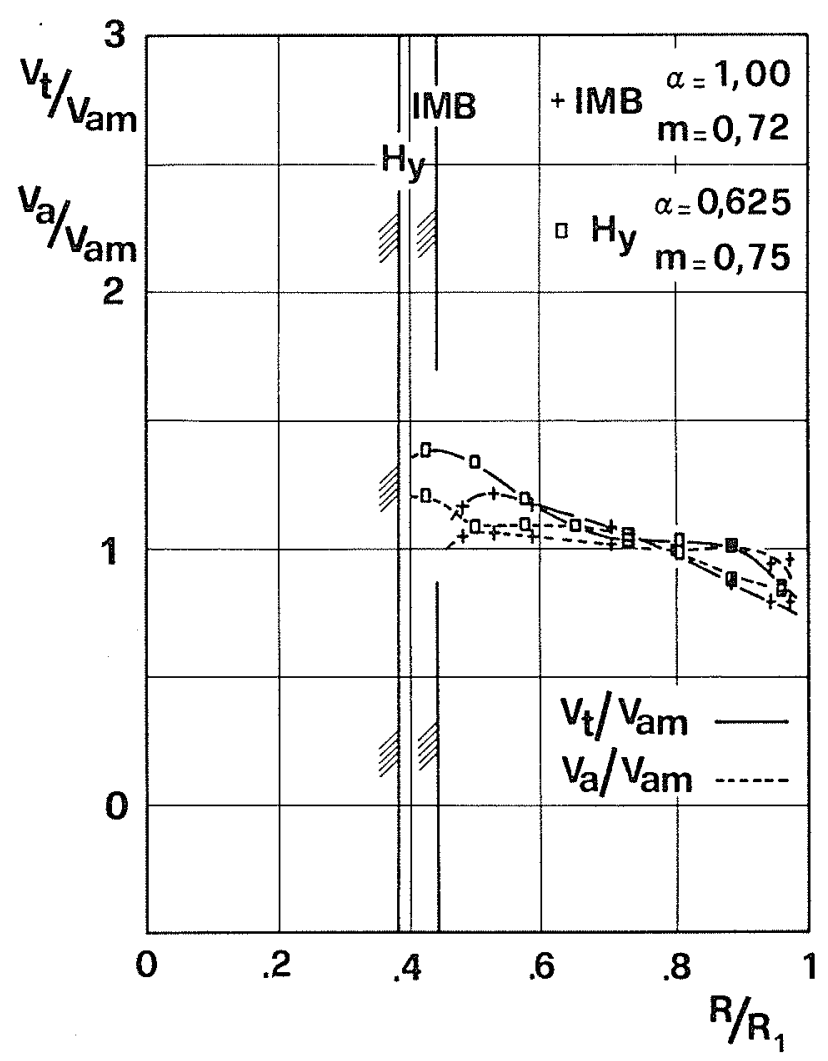

Figure 6

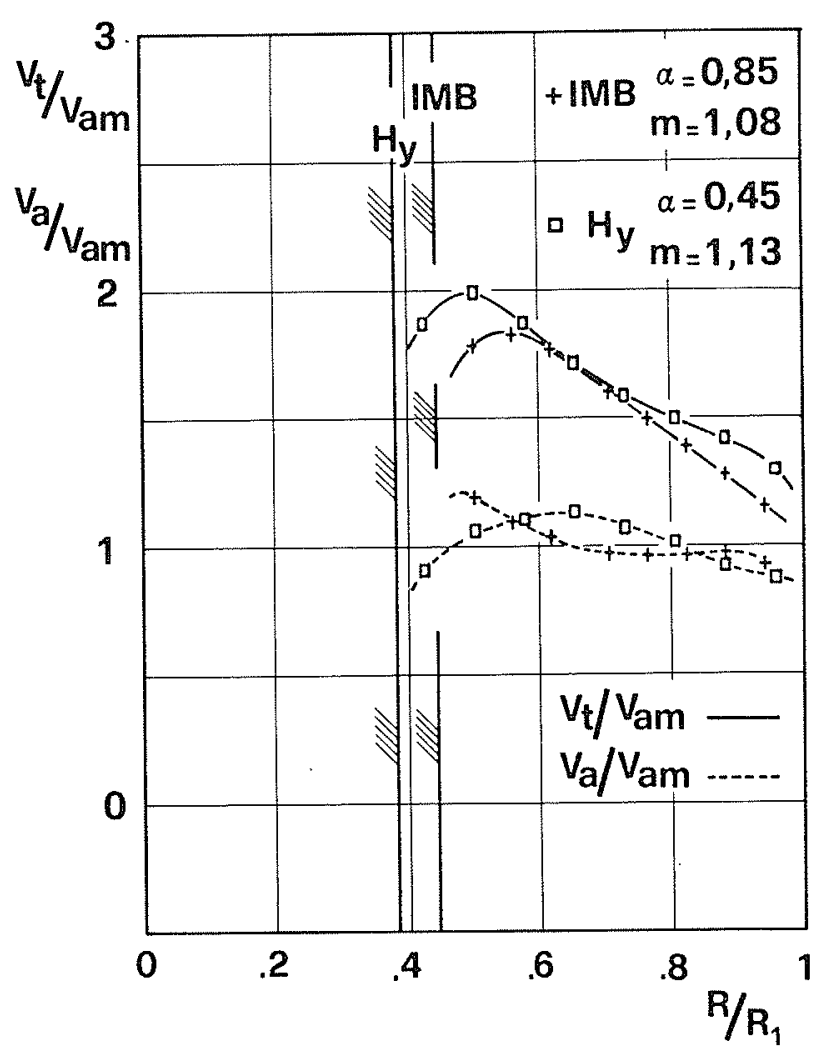

Figure 7

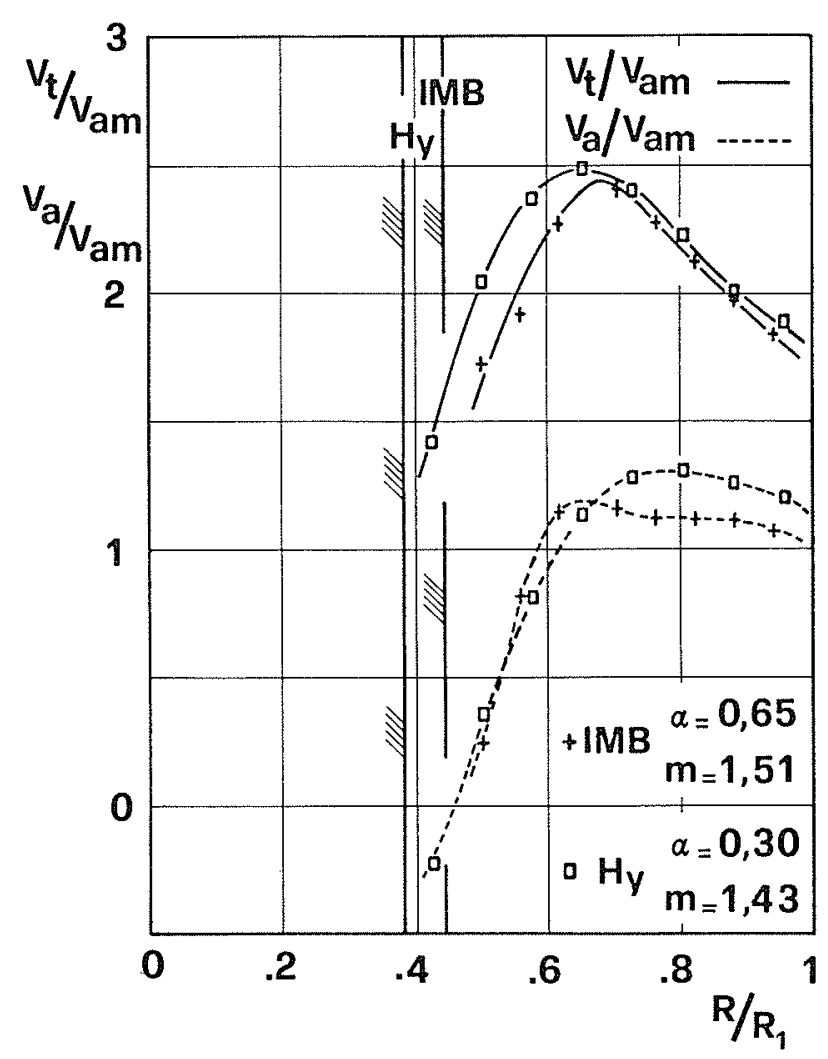

Figure 8

Figures 5, 6, 7, 8 - Comparaison des vitesses dans la zone axiale des deux canaux pour des valeurs comparables du paramètre $m$. 
L'analogie de l'évolution de l'écoulement dans les deux canaux est davantage mise en évidence en comparant les distributions de vitesse ayant le même paramètre $m$, appelé "swirl rate", généralement employé dans la litérature pour évaluer l'intensité tourbillonnaire de la veine fluide [6], [7].

Le paramètre $m$ est défini comme le rapport entre le flux du moment de la quantité de mouvement tangentiel et le flux de la quantité de mouvement axial multiplié par le rayon extérieur du canal :

$$
m=\frac{\int_{R_{0}}^{R_{1}} 2 \pi \rho V_{t} V_{a} R^{2} d R}{R_{1} \int_{R_{0}}^{R_{1}} 2 \pi \rho V_{a}^{2} R d R}
$$

Dans les figures de 5 à 8 , on compare directement les distributions des vitesses pour lesquelles les valeurs de $m$ se rapprochent l'une de l'autre.

Pour les valeurs de $m$ inférieures à 0,75 , près du moyeu, on ne remarque dans aucun des deux canaux le phénomène décrit précédemment (fig. 5 et 6 ). Ce phénomène commence à des valeurs de $m$ voisines de 1 , comme le montre la fig. 7 où l'on remarque une bonne analogie des composantes tangentielles de vitesse, même s'il existe des différences dans les répartitions des composantes axiales.

Pour une valeur de $m \simeq 1,5$ le noyau central à vitesse axiale faible ou négative et à tourbillon presque forcé est mis en évidence dans les deux canaux (voir fig. 8) et les allures des composantes axiales et tangentielles de vitesse sont analogues.

Evaluation des dimensions de la zone centrale à vitesse axiale faible ou négative et à tourbillon presque forcé

La comparaison des distributions de vitesse relevées dans les sections IMB2 et $\mathrm{HyG}$ montre clairement que, malgré des différences dans la géométrie des canaux et la nature du fluide d'essai,pour des vakeurs élevées de $m$ se forme un noyau central, coaxial au moyeu, appelé par quelques auteurs "noyau d'eau morte" [1-2], dans lequel on constate des anomalies dans la distribution des vitesses.

On peut introduire plusieurs grandeurs géométriques pour déterminer la zone affectée par les phénomènes qu'on examine. En [1] et [2] on utilise le rayon $r_{i}$ basé sur la distribution de la vitesse axiale calculée en supposant que tout le débit s'écoule uniquement au travers de la couronne annulaire extérieure à $r_{i}$.

Au contraire en [3] on propose le rayon du noyau central ̀̀ tourbillon forcé $r_{f}=\sqrt{K / \omega}$ basé sur une schématisation de la distribution des composantes tangentielles de vitesse qui suppose un tourbillon libre $V_{t}=K / \mathrm{R}$ dans une couronne annulaire extérieure et un tourbillon forcé $V_{t}=\omega R$ dans le noyau central : la constante du tourbillon libre $K$ et la rotation $\omega$ du tourbillon forcé sont déterminées à partir des résultats expérimentaux.

Le diagramme de fig. 9 indique les allures de $r_{f}$ et $r_{i}$ en fonction du paramètre.

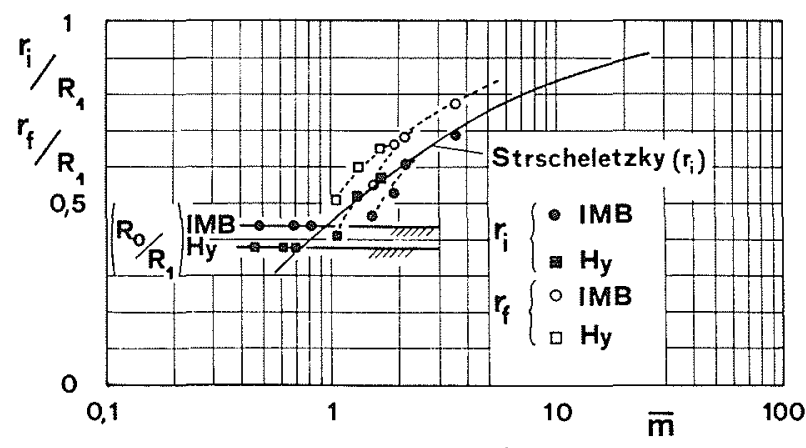

Figure 9 - Rayon du "noyau d'eau morte" $r_{i}$ et du "noyau à tourbillon forcé" $r_{f}$ en fonction du paramètre $\bar{m}$.

$$
\bar{m}=\frac{R_{1}}{2 B}\left[1-\left(\frac{R_{0}}{R_{1}}\right)^{2}\right] \operatorname{tang} \gamma
$$

qui a l'avantage, par rapport à $m$, de dépendre seulement des paramètres géométriques du conduit : $\mathrm{B}$ hauteur du distributeur, $R_{1}$ et $R_{0}$ respectivement rayon extérieur et intérieur de la section considérée et $\gamma$ angle formé à la sortie du distributeur par la ligne moyenne du conduit entre les aubes et la direction radiale (voir fig. 2). Il coincide avec le paramètre $m$ défini par la relation (1) si l'on introduit les hypothèses simplificatrices suivantes :

- distribution uniforme de la vitesse axiale dans la section de mesure,

- vitesse radiale uniforme sur toute la hauteur du distributeur,

- pertes négligeables entre la sortie du distributeur et la section de mesure,

- angle de sortie du fluide coïncident avec l'angle des aubes du distributeur.

Les valeurs de $\bar{m}$ calculées au moyen des paramètres géométriques par la relation (2) et celles de $m$ obtenues en utilisant les résultats expérimentaux sont comparées dans le diagramme de la fig. 10 en fonction de $\alpha$.

Pour le canal IMB les valeurs expérimentales sont toujours au-dessous de la courbe théorique; pour le canal $\mathrm{Hy}$, au contraire, l'écart entre les valeurs expérimentales et la courbe théorique n'est pas toujours dans le même sens. Cette différence peut être imputée à des différences d'alimentation des directrices (pour IMB, alimentation par chambre libre et pour $\mathrm{Hy}$, alimentation par bâche spirale et avant directrices) et à la position des aubes du distributeur (pour IMB, en zone à écoulement essentiellement radial, pour $\mathrm{Hy}$, en zone à écoulement mixte).

Le paramètre $\bar{m}$ peut être utilisé comme indice de la présence du phénomène de recirculation dans un conduit axial à section annulaire alimenté par un distributeur radial et permet (comme l'on peut voir sur la fig. 9) de déterminer les rayons $r_{i}$ et $r_{f}$, même de schématiser les répartitions respectivement des vitesses axiales et tangentielles aux petites ouvertures du distributeur.

Les différences des valeurs de $r_{i}$ et de $r_{f}$ entre les deux canaux peuvent être imputées à la géométrie des conduits et surtout à la différence du rapport $D_{0} / D_{1}$ qui détermine l'amorçage du phénomène de recirculation pour différentes valeurs de $\bar{m}$. 


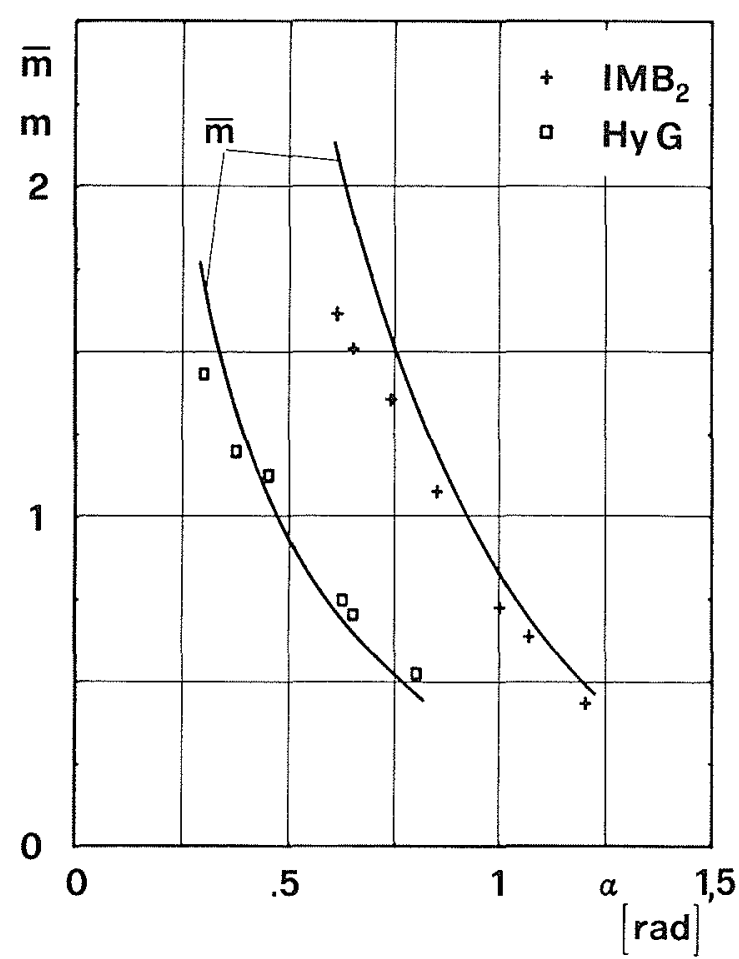

Figure 10 - Comparaison entre les valeurs expérimentales et géométriques de "swirl rate".

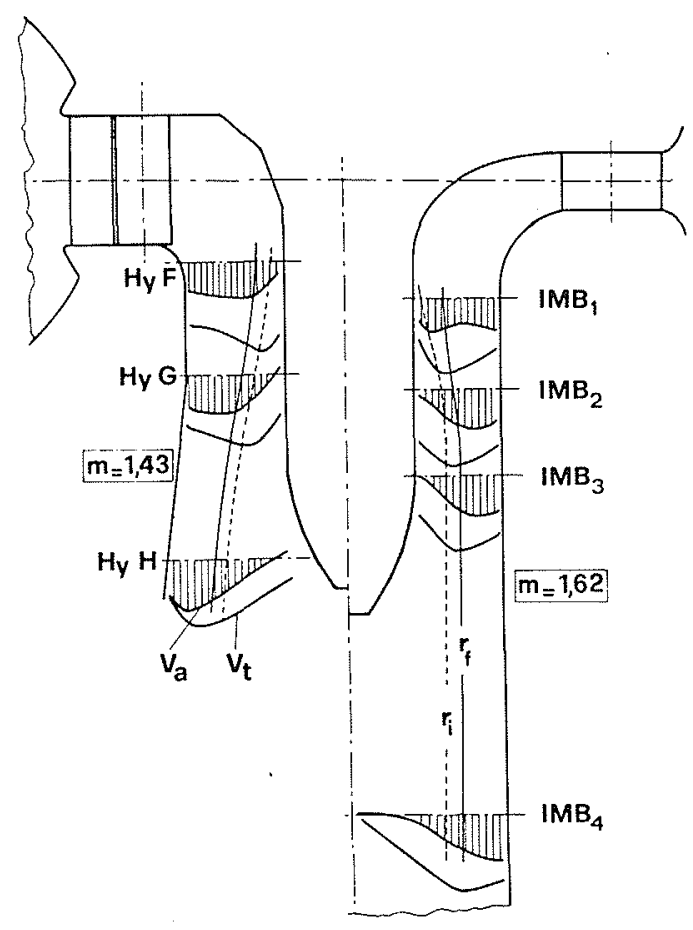

Figure 11 - Evolution axiale du "noyau d'eau morte" et du "noyau à tourbillon forcé" dans les deux canaux.

En outre, il faut remarquer que les deux sections de mesure ne sont pas placées exactement dans la même position axiale et que les répartitions de vitesse se ressentent de ce fait, comme l'on peut le voir sur la fig. 11 où est indiqué, le long des deux canaux, un exemple d'évolution des composantes axiales et tangentielles de la vitesse, de $r_{i}$ et $r_{f}$, avec $m=1,43$ pour $\mathrm{Hy}$ et $m=1.62$ pour IMB.
La figure montre que le noyau d'eau morte se développe essentiellement dans la zone axiale, en étendant rapidement son champ d'influence et en se stabilisant ensuite sur une aire pratiquement constante.

Sur la fig. 9, les valeurs de $r_{i}$ déterminées par les relevés expérimentaux sont comparées à celles de la courbe théorique de Strscheletzky obtenue pour des conduits axiaux à section circulaire sans destructeur de tourbillon en aval, sur la base de la théorie de l'équilibre cinétique des courants de fluides incompressibles, homogènes, non-visqueux [1]. Les écarts majeurs sont relevés pour les valeurs de $\vec{m}$ pour lesquelles commence, au voisinage du moyeu, le phénomène "d'eau morte".

Effets du "noyau d'eau morte" sur le fonctionnement de la turbine

Les mesures de vitesse décrites jusqu'ici ont été effectuées sans roue.

Des essais effectués dans le canal du modèle Hy d'origine ont montré qu'aux ouvertures élevées du distributeur, au moins jusqu'à la section $F$, l'écoulement est indépendant de la présence de la roue.

Aux petites ouvertures, même en présence de la roue, il se forme un noyau central à tourbillon presque forcé avec diminution des pressions totales et des vitesses méridiennes, semblable à celui précédemment décrit dans le cas sans roue; comme on peut voir sur la fig. 12 pour la section $F$, la présence de la roue, tout en modifiant la

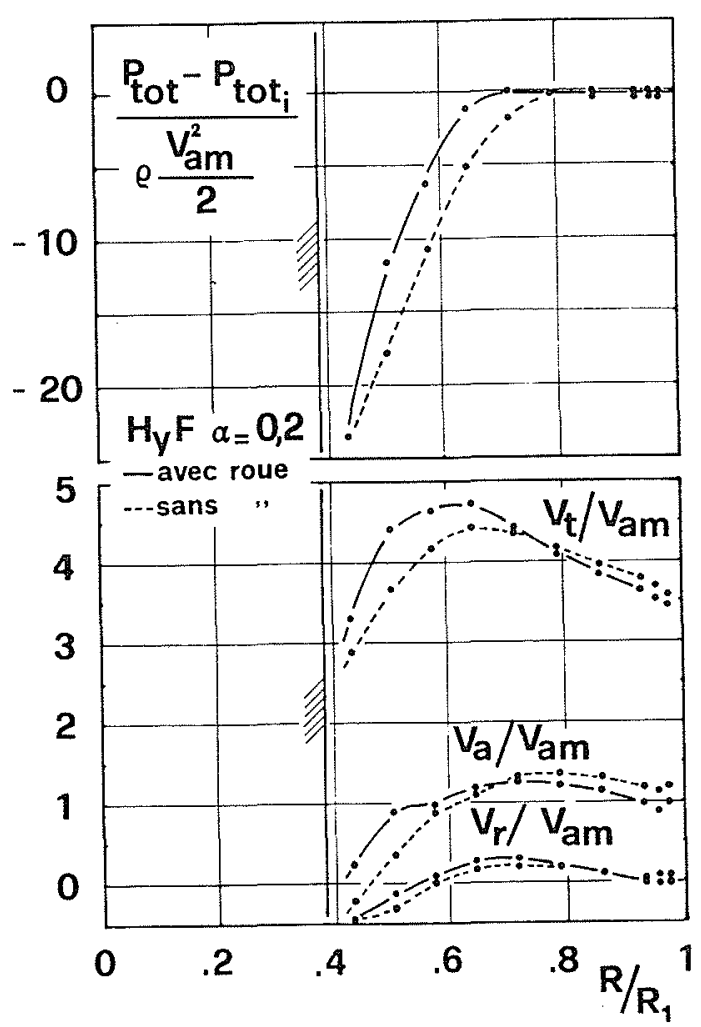

Figure 12 - Comparaison des vitesses et pressions totales avec et sans roue. 

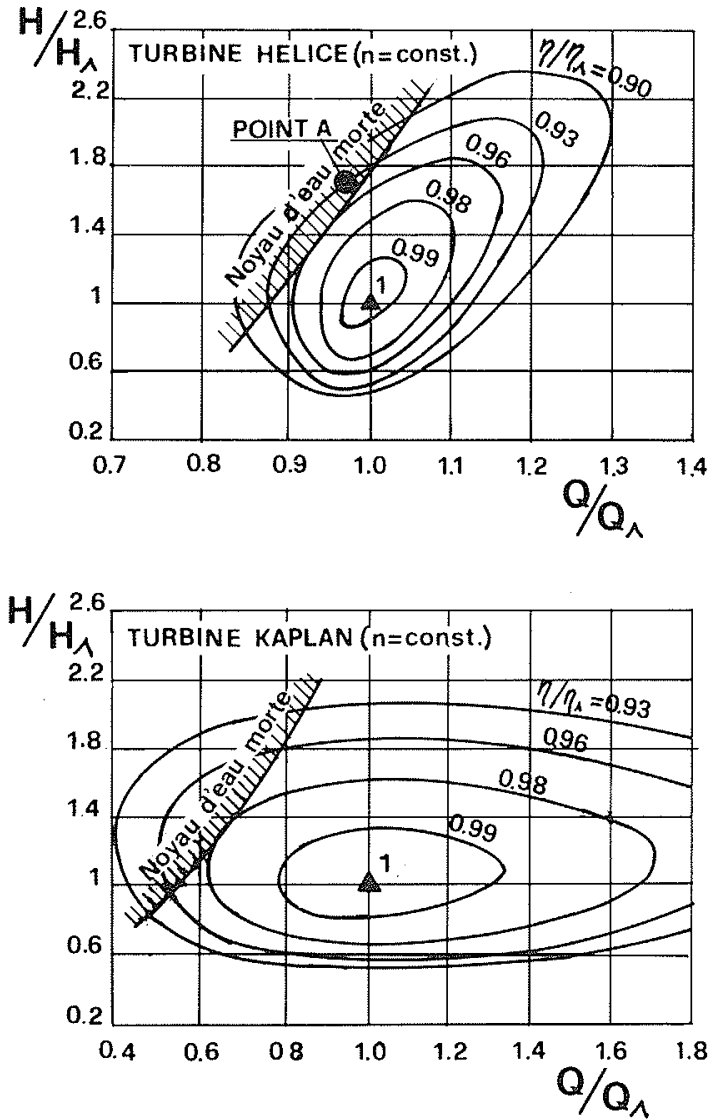

Figure 13 - Zones affectées par le "noyau d'eau morte" sur les diagrammes des rendements en colline d'une turbine-hélice et d'une turbine Kaplan.

distribution de vitesse et de pression totale à l'intérieur du noyau central, ne change pas les caractéristiques générales du phénomène et diminue légèrement les dimensions de la zone intéressée.

Les résultats de l'analyse effectuée dans les paragraphes précédents, en ce qui concerne le rayon limite du noyau central, restent donc valables en première approximation pour des canaux adducteurs de turbines axiales, bien qu'il soit nécessaire d'examiner plus précisément l'altération apportée par la roue sur les distributions de vitesse et de pression effectives à l'intérieur du noyau central.

Analysons maintenant les effets du "noyau d'eau morte" sur le fonctionnement de la turbine.

Les zones de fonctionnement intéressées par le phénomène sont indiquées (fig. 13) sur les diagrammes en colline d'une turbine-hélice et d'une turbine Kaplan de vitesse spécifique moyenne. Dans ces zones, la présence du noyau central d'eau morte est une des causes de la baisse du rendement et agit suivant deux mécanismes de perte. En effet, il y a des pertes liées à la formation du noyau central, comme le montrent les chutes de pression totale relevées en amont de la roue (fig. 12), et des pertes dues aux interactions entre le noyau central et la roue, liées à la formation, près du moyeu, d'angles d'incidence de l'écoulement sur les aubes très différentes des angles d'incidence optimaux pour lesquels les aubes ont été projetées.

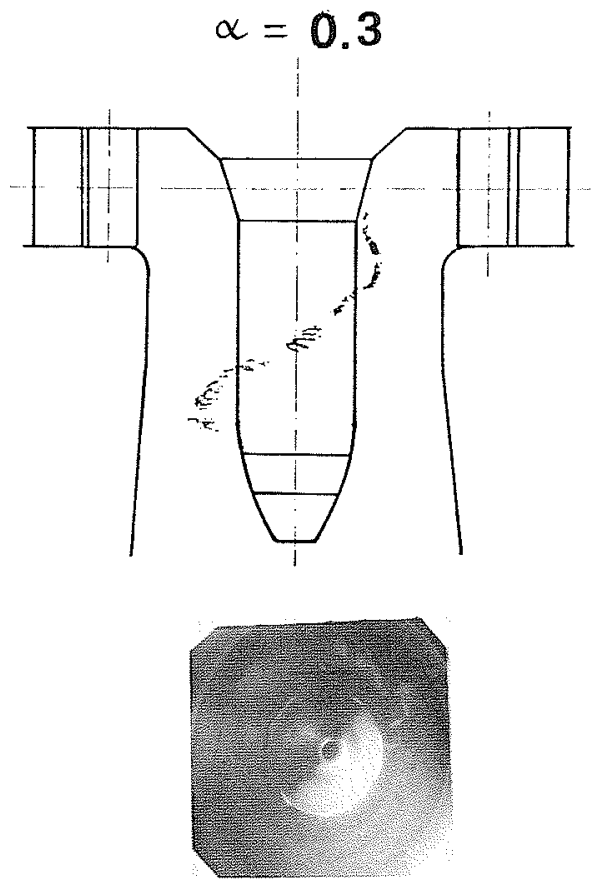

Figure 14 - Tourbillon cavitant dans le canal sans roue.

POINT A (v.Fig.13) : $\alpha=0.3$
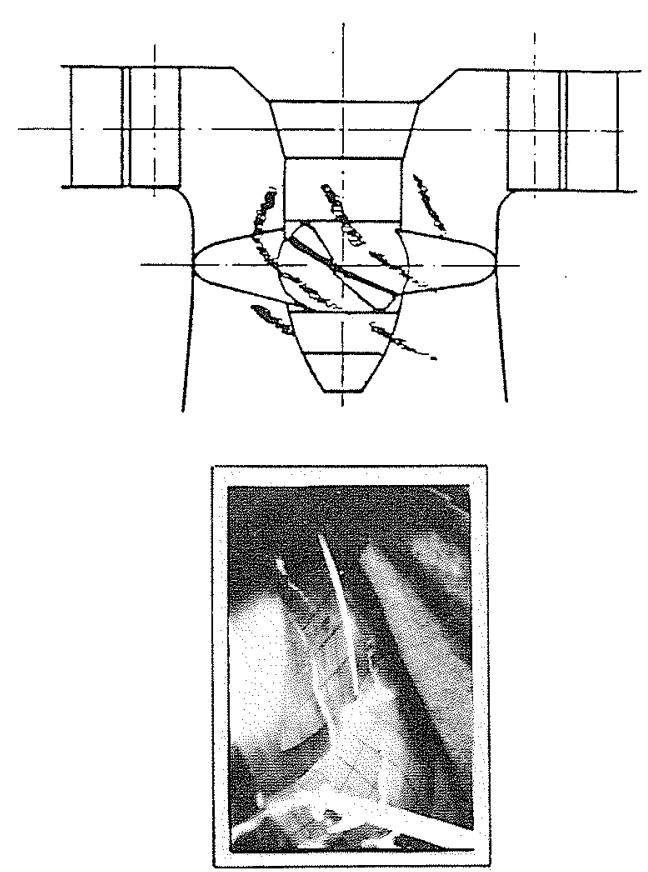

Figure 15 - Tourbillons cavitants entre les aubes de la roue.

En ce qui concerne l'influence du phénomène sur le comportement à cavitation de la machine, il faut remar quer qu'aux petites ouvertures du distributeur, même pour des valeurs élevées du coefficient de cavitation, près du moyeu, dans l'espace entre distributeur et roue, se forment des tourbillons cavitants intermittents qui, comme dans le cas sans roue (fig. 14), se détachent de la zone d'eau morte et passent au travers de la roue (fig. 15), en créant des vibrations et des bruits aux basses charges de fonctionnement. 


\section{Symbolique}

$B$ hauteur du distributeur

$D_{1}$ diamètre extérieur du conduit

$D_{0}$ diamètre du moyeu

$H$ chute

$K \quad$ flux du moment de la quantité de mouvement par unité de débit

$m$ "swirl rate" déduit des résultats expérimentaux

$\bar{m}$ "swirl rate" calculé à partir des paramètres géométriques du conduit

$n \quad$ vitesse de rotation

$P_{\text {tot }}$ pression totale

$P_{\text {tot }_{\mathrm{i}}}^{\text {pression totale à l'entrée de la bache spirale }}$

$Q$ débit

$R$ rayon

$r_{f} \quad$ rayon du "noyau à tourbillon forcé"

$r_{i}$ rayon du "noyau d'eau morte"

$R e \quad$ nombre de Reynolds $=\frac{V D_{\text {hydraulique }}}{v}$
$V \quad$ vitesse

$Z_{d} \quad$ nombre des aubes du distributeur

$\alpha \quad$ angle d'ouverture des aubes du distributeur référé à la position de fermeture [rad]

$\gamma \quad$ angle formé à la sortie du distributeur par la ligne moyenne du conduit entre les aubes et la direction radiale

$\eta \quad$ rendement

$\rho \quad$ densité

$\omega$ rotation

\section{Indices}

a axial

$m$ moyen

$t \quad$ tangentiel

$\Lambda \quad$ situation de rendement maximum

\section{Bibliographie}

[1] STRSCHELETZKY M. - Gleichgewichtsformen der rotationssymmetrischen Strömungen mit konstantem Drall in geraden, zylindrischen Rotationshohlräumen. Voith Forschung und Konstruktion, Octobre 1959.

[2] SCHLUNKES F. - Messungen an Luftströmungen mit konstantem Drall in geraden Kreisrohr. Voith - Forschung und Konstruktion, Octobre 1959

[3] BETTOCCHI R., CANTORE G. - Ricerche sperimentali sul flusso in canali rettilinei di sezione circolare in uscita da un distributore cilindrico. XXXVII Congrès National A.T.I. Padoue, Septembre 1982.
[4] EICHLER O. - Vibration phenomena on hydraulic axial turbines. IAHR/IUTAM 1979 - Karlsruhe.

[5] BETTOCCHI R., CANTORE G. - Ricerche sperimentali sul flusso vorticoso in un canale rettilineo di sezione anulare. XXXVII Congrès National A.T.I. Padoue, Septembre 1982.

[6] NISHI M., KUBOTA T., MATSUNAGA S., SENOO Y. Study on swirl flow and surge in an elbow type draft tube. IAHR Symposium 1980 - Tokyo.

[7] SENOO Y., NAGATA T. - Swirl flow in long pipes with different roughness. Bulletin JSME $15-90$ (1972) 1514.

Appendice au mémoire : Analyse de l'écoulement dans la zone axiale des canaux adducteurs des turbines-hélices"

présentée par M.L. Magri

Dans le cadre des recherches sur les mini-turbines axiales tubulaires standard (T.A.T.), dont une des versions de base est représentée dans la fig. 1 avec une roue à 5 aubes ayant une vitesse spécifique au point de rendement maximal égale à $n_{q \wedge}=n Q_{\wedge}^{0,5} / H_{\wedge}^{0,75}=130$, Hydroart a essayé dans ses laboratoires deux versions $\mathrm{A}$ et $\mathrm{B}$ d'un distributeur à aubes fixes à simple courbure

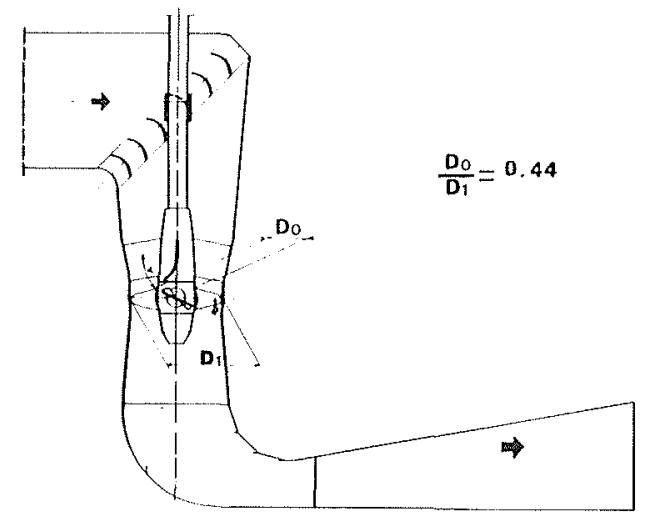

Figure 1. - Turbine axiale tubulaire T.A.T. et épaisseur constante (fig. 2) qui diffèrent par la corde et l'angle de sortie des aubes.

Les valeurs correspondantes du paramètre $\bar{m}$, en considérant $\alpha$ et la vitesse moyenne constants dans la section $X$ (voir fig. 2), sont pour les deux cas : $\bar{m}_{A}=1,2 ; \bar{m}_{B}=0,89$.

On peut observer que pour une valeur du rapport
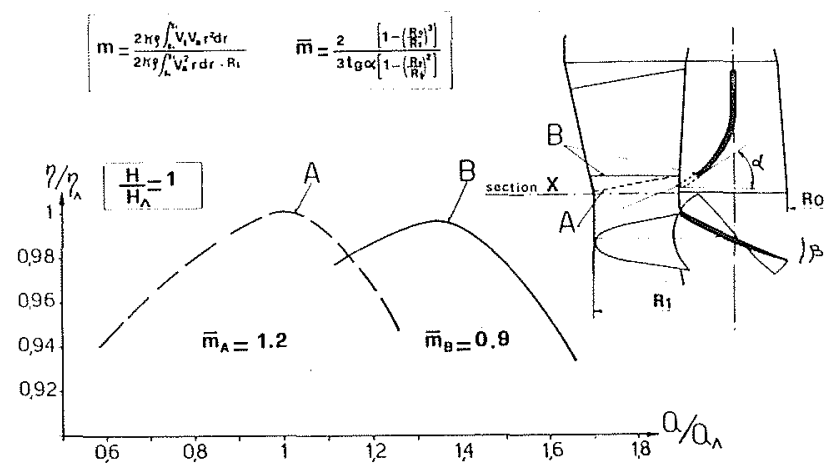

Figure 2. - T.A.T. avec aubes de la roue mobiles. 


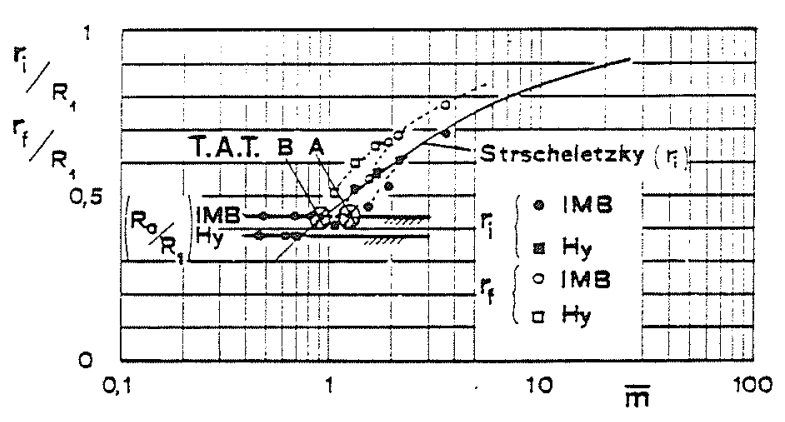

Figure 3. - Rayons du noyau d'eau morte $f(\bar{m})$.

des rayons $R_{0} / R_{1}=0,44$, les valeurs $\bar{m}_{B}$ et $\bar{m}_{A}$ se trouvent respectivement sur la courbe limite théorique proposée par Strscheletzky $r_{i}$ et à droite de celle-ci. (fig. 3).
D'après les résultats que nous possédons nous pouvons affirmer que, dans tout le champ exploré, on n'a pas trouvé la moindre présence de phénomènes tourbillonnaires même lorsqu'on diminue le sigma d'essai pour en faciliter la visualisation $(\sigma=1,5 \div 0,25)$.

Ceci confirme ce qui a déjà été reporté dans le mémoire sur la fig. 9 où l'on relève le début du phénomène de noyau d'eau morte pour $\bar{m}=1,1$ avec un rapport moyeu-manteau $R_{0} / R_{1}=0,38$ (Hy) et pour $\bar{m}=1,5$ avec un rapport moyeu-manteau $R_{0} / R_{1}=0,44$ (IMB). On constate donc que la courbe limite $r_{i}$, qui signale le début du noyau d'eau morte d'où se détachent les filaments tourbillonnaires dans le cas de canaux cylindriques annulaires, est déplacée vers des valeurs de $\bar{m}$ plus grandes (à rapport $R_{0} / R_{1}$ égal) que les valeurs théoriques relatives aux canaux circulaires simples: ce déplacement devrait être vérifié systématiquement d'une façon expérimentale en tenant compte de la présence de la roue.

\section{Discussion}

Président : R. BALLAY

Après avoir remercié les auteurs pour leur contribution à la compréhension et à la prévision des fonctionnements à débit partiel des turbines, M. le Président demande si les résultats sont transposables du modèle au prototype.

MM. CANTORE et MAGRI précisent que la transposition des résultats est bien valable en ce qui concerne tous les phénomènes qui sont régis par les lois de la similitude hydraulique.
L'on pourra à l'avenir faire des mesures sur des machines industrielles pour bien maîtriser la transposition dans le cas où il y a formation des tourbillons cavitants.

M. le Président clôture la séance en remerciant tous les participants.

\section{Abstract \\ Experimental analysis of the flow in the axial region of propeller-turbine admission ducts}

The paper presents some results of the measurements carried out, in water, in the axial region of a propeller turbine admission duct (Hy), without runner, and, in air, in an annular section cylindrical duct fed by a radial distributor (IMB) (see Fig. 2). The measurements have been performed with different wicket-gate openings $\alpha$ and the attention has been drawn on the small openings where different phenomena have been observed (see Fig. 1):

- a variation of the radial distribution of the tangential velocity near the hub, associated with the arising of a quite forced vortex;

- the decrease and sometimes the inversion of the axial velocity;

- the presence of radial flows directed towards the hub;

- the drop of the total pressure, due to the viscous dissipation

These phenomena mainly develop in the axial region of the two ducts, rapidly extend their influence and finally settle on a practically constant area (see Fig. 11).

The distribution of axial and tangential velocity components change almost similarly in the two ducts according to the decrease of the wicket-gate opening, as it can be observed in figures 3 and 4.

These figures show the distribution of axial and tangential velocity components divided by the average axial velocity, as a function of the ratio between the radius of the measurement points and the external one in the closest sections of the two ducts (IMB 2 and $\mathrm{HyG}$ ).
The similarity of the flow evolution in the two ducts is even more underlined by the comparison (see Fig. 5-8) of the velocity distributions for comparable values of the $m$ parameter, called swirl rate, defined by the equation (1) and usually utilized in technical literature for the evaluation of the flow vortex intensity.

In the diagram of figure 9 the radius limiting the central core, which is characterized by the decrease of axial velocity $\left(r_{i}\right)$ and the radius of the core characterized by the quite forced vortex distribution of tangential velocities $\left(r_{f}\right)$ have been associated to $\bar{m}$ parameter values, calculated by means of the geometric data of the duct according to the equation (2). The $\bar{m}$ and $m$ values have been compared in the diagram of figure 10 versus $\alpha$.

Some experimental tests carried out on the Hy model with runner showed that, at small openings, a central core similar to the one previously described in the case without runner is developed (see Fig. 12).

In axial turbines this phenomenon causes efficiency drop and cavitating vortexes which, as it happens without the runner (see Fig. 14), detach near the hub and pass through the runner (see Fig. 15) in a helix spiral form, thus causing vibrations and noise at low operation loads.

The operation areas concerned by this phenomenon are indicated in figure 13 on the hill diagrams of a propeller and a Kaplan turbine with medium specific speed. 